\title{
Adenomatoid tumors of the female and male genital tracts: a clinicopathological and immunohistochemical study of 44 cases
}

\author{
Ankur R Sangoi, Jesse K McKenney, Erich J Schwartz, Robert V Rouse and Teri A Longacre \\ Department of Pathology, Stanford University, Stanford, CA, USA
}

\begin{abstract}
Adenomatoid tumors of the female and male genital tracts are well characterized as mesothelial in origin, but a detailed histological and immunohistochemical analysis comparing both traditional and newer mesothelial markers across gender and site has not been formally conducted. A variety of morphologic features previously described as characteristic of adenomatoid tumors were evaluated in $\mathbf{4 4}$ adenomatoid tumors from the male and female genital tracts. Immunohistochemical analysis with pankeratin (AE1/CAM5.2), WT-1, calretinin, CK5/6, D240 , and caldesmon was also performed. The extent and intensity of staining were scored semiquantitatively on one representative section per case and mean value for each parameter was calculated. All $(n=44)$ the adenomatoid tumors from both the female and male genital tracts demonstrated a distinctive thread-like bridging strand pattern. Lymphoid aggregates were seen in all 12 adenomatoid tumors of male patients, but in only 4 of $32(13 \%)$ tumors in female patients $(P<0.0001)$. The remaining morphologic features were variably present with no clear sex predilection. Pankeratin, calretinin, and D2-40 reactivity were identified in all female $(n=32)$ and male $(n=12)$ genital tract adenomatoid tumors. Adenomatoid tumors expressed WT-1 in 11/12 (92\%) male patients and in $31 / 32(97 \%)$ female patients. In male patients, reactivity for CK5/6 and caldesmon was found in $1 / 12(8 \%)$ and $0 / 12(0 \%)$ adenomatoid tumors (respectively), whereas reactivity in female patients was found in $5 / 32(16 \%)$ and $1 / 32(3 \%)$; respectively. Female tumors differ from their male counterparts by the frequent absence of lymphoid aggregates and the presence of a circumscribed margin when occurring in the fallopian tube. Of the putative mesothelial markers evaluated, calretinin, D2-40, and WT-1 show a similar immunoprofile and have a higher sensitivity than CK5/6 and caldesmon in genital tract adenomatoid tumors. However, the presence of additional, often strong expression of WT-1 in normal tissues of the female genital tract limits the utility of WT-1 in this setting.
\end{abstract}

Modern Pathology (2009) 22, 1228-1235; doi:10.1038/modpathol.2009.90; published online 19 June 2009

Keywords: adenomatoid tumor; genital tract; calretinin; D2-40; WT-1; caldesmon

Although extragenital adenomatoid tumors have been reported in the adrenal gland, appendix, heart, hernia sac, intestinal mesentery, liver, lymph node, mediastinum, omentum, pancreas, peritoneum, pleura, and umbilicus, ${ }^{1-12}$ they have been characteristically described within genital tract sites of both male and female patients. Initially designated as 'benign mesothelioma of the genital tract' in 1942 by Masson et $a l,{ }^{13}$ the term 'adenomatoid tumor' was

Correspondence: Dr AR Sangoi, MD, Department of Pathology, Stanford University School of Medicine, 300 Pasteur Drive, Room L-235, Stanford, CA, USA.

E-mail: asangoi2@yahoo.com

Current address: AR Sangoi, El Camino Hospital, Department of Pathology, 2500 Grant Road, Mountain View, CA 94039, USA.

Presented in part at the 98th meeting of the United States and Canadian Academy of Pathology, Boston, MA, March 2009

Received 12 February 2009; revised 14 May 2009; accepted 16 May 2009; published online 19 June 2009 coined in 1945 by Golden and $\mathrm{Ash}^{14}$ to denote these benign, often incidental, and typically well-circumscribed neoplasms of mesothelial origin. ${ }^{12,15-35} \mathrm{~A}$ variety of immunohistochemical markers have been reported to identify adenomatoid tumors, but a detailed histological and immunohistochemical study comparing both traditional and newer mesothelial markers has not been previously conducted. The aim of this study was to evaluate the histological and immunohistological features of a series of female and male genital tract adenomatoid tumors to further refine their characteristics and determine whether there are gender or site specific differences.

\section{Materials and methods}

A total of 44 adenomatoid tumors from the female and male genital tracts were retrieved from the 
Table 1 Antibody sources and dilutions

\begin{tabular}{lllll}
\hline Antibody & Clone & Dilution & Antigen retrieval & Source \\
\hline Caldesmon & h-CD & $1: 50$ & None & Dako (Carpinteria, CA, USA) \\
Calretinin & Polyclonal & $1: 100$ & Benchmark standard & Cell Marque (Rocklin, CA, USA) \\
CK5/6 & D5/16 B4 & $1: 200$ & Benchmark standard & Zymed (San Francisco, CA, USA) \\
D2-40 & D2-40 & $1: 80$ & None & Covance (Princeton, NJ, USA) \\
Pankeratin & AE1/AE3; CAM5.2 & $1: 50 ; 1: 50$ & Protease 2 & Dako; Becton Dickinson (San Jose, CA, USA) \\
WT-1 & 6F-H2 & $1: 400$ & None & Dako
\end{tabular}

Table 2 Clinicopathological features of female and male genital tract adenomatoid tumors

\begin{tabular}{lcccc}
\hline Site & Incidental (\%) & Age (years); range (mean) & Size (cm); range (mean) & Laterality (\%); $R=$ right \\
\hline Fallopian tube $(n=4)$ & 100 & $38-51(43)$ & $0.1-0.4(0.2)$ & $\mathrm{R}=50$ \\
Myometrium $(n=26)$ & 65 & $26-65(44.4)$ & $0.4-2.7(1.6)$ & $\mathrm{N} / \mathrm{A}$ \\
Ovary $(n=2)$ & 50 & $77-79(78)$ & $0.2-1.6(0.9)$ & $\mathrm{R}=50$ \\
Epididymis $(n=8)$ & 0 & $32-66(46.6)$ & $0.7-2(1.6)$ & $\mathrm{R}=63$ \\
Paratestis $(n=4)$ & 0 & $38-71(54)$ & $0.8-1.5(1)$ & $\mathrm{R}=25$ \\
† overall $(n=32)$ & 68 & $26-77(46.3)$ & $0.1-2.4(1.4)$ & $\mathrm{R}=50$ \\
ơ overall $(n=12)$ & 0 & $32-71(49.3)$ & $0.7-2(1.1)$ & $\mathrm{R}=50$ \\
\hline
\end{tabular}

Table 3 Histologic features of female and male genital tract adenomatoid tumors

\begin{tabular}{|c|c|c|c|c|c|}
\hline Site & $\begin{array}{c}\text { Infiltrative } \\
(\%)\end{array}$ & $\begin{array}{l}\text { Signet-ring } \\
\text { cells }(\%)\end{array}$ & $\begin{array}{l}\text { Vacuolated } \\
\text { cells (\%) }\end{array}$ & $\begin{array}{l}\text { Thin bridging } \\
\text { strands }(\%)\end{array}$ & $\begin{array}{c}\text { Lymphoid } \\
\text { aggregates (\%) }\end{array}$ \\
\hline Fallopian tube $(n=4)$ & 0 & 100 & 75 & 100 & 50 \\
\hline Myometrium $(n=26)$ & 85 & 100 & 88 & 100 & 8 \\
\hline Ovary $(n=2)$ & 50 & 50 & 100 & 100 & 0 \\
\hline Epididymis $(n=8)$ & 86 & 100 & 100 & 100 & 100 \\
\hline Paratestis $(n=4)$ & 75 & 100 & 75 & 100 & 100 \\
\hline o overall $(n=32)$ & 72 & 97 & 88 & 100 & 13 \\
\hline o overall $(n=12)$ & 82 & 100 & 91 & 100 & 100 \\
\hline
\end{tabular}

pathology archives of Stanford University Medical Center and Veterans Affairs Palo Alto Health Care System. Each case was reviewed by three of the authors (ARS, JKM, and TAL) and diagnoses for all cases were confirmed by routine hematoxylin and eosin staining. One case originally diagnosed as paratesticular adenomatoid tumor was reclassified as adenomatoid hyperplasia of rete testis and, therefore, excluded. Immunohistochemical staining using antibodies against pankeratin, calretinin, D2-40, WT-1, and caldesmon was performed on one representative section per case on 4-mm thick formalin-fixed, paraffin-embedded sections mounted on charged slides and baked at $60^{\circ} \mathrm{C}$ for $1 \mathrm{~h}$. The primary antibodies used in the study are listed in Table 1. All cases were stained in parallel with appropriate positive and negative controls. Staining extent was semiquantitatively scored as negative ( $0,<5 \%$ cells stained), focally positive $(1+, 5-10 \%$ cells stained), positive $(2+, 10-50 \%$ cells stained), or diffusely positive $(3+,>50 \%$ cells stained), and a mean extent (ME; range 0-3) calculated. Staining intensity was semiquantitatively scored from 0 to 3 + and a mean intensity (MI; range 0-3) calculated. In addition to an assessment of clinicopathologic features, morphologic differences of adenomatoid tumor in female versus male sites, if any, were compared including features previously described as characteristic of adenomatoid tumor. Statistical analysis utilized Fisher's two-tailed exact test, with significance set at $P<0.05$.

\section{Results}

\section{Clinical Features}

Comparative clinicopathological features of female and male genital tract adenomatoid tumors are listed in Table 2. Among the adenomatoid tumors within the female genital tract, the myometrium was the most frequent site involved, constituting 26 of the 32 $(81 \%)$ cases. In the male genital tract, adenomatoid tumors were more frequently seen in the epididymis compared to the testis/paratestis (75 versus $25 \%$ ). All 12 male adenomatoid tumors presented as mass lesions, whereas female genital tract tumors were more often incidental findings $(68 \%)$ discovered on microscopic examination of specimens removed for diagnoses of uterine leiomyomata, adenomyosis, ovarian carcinoma, ovarian abscess, tubal ligation, 


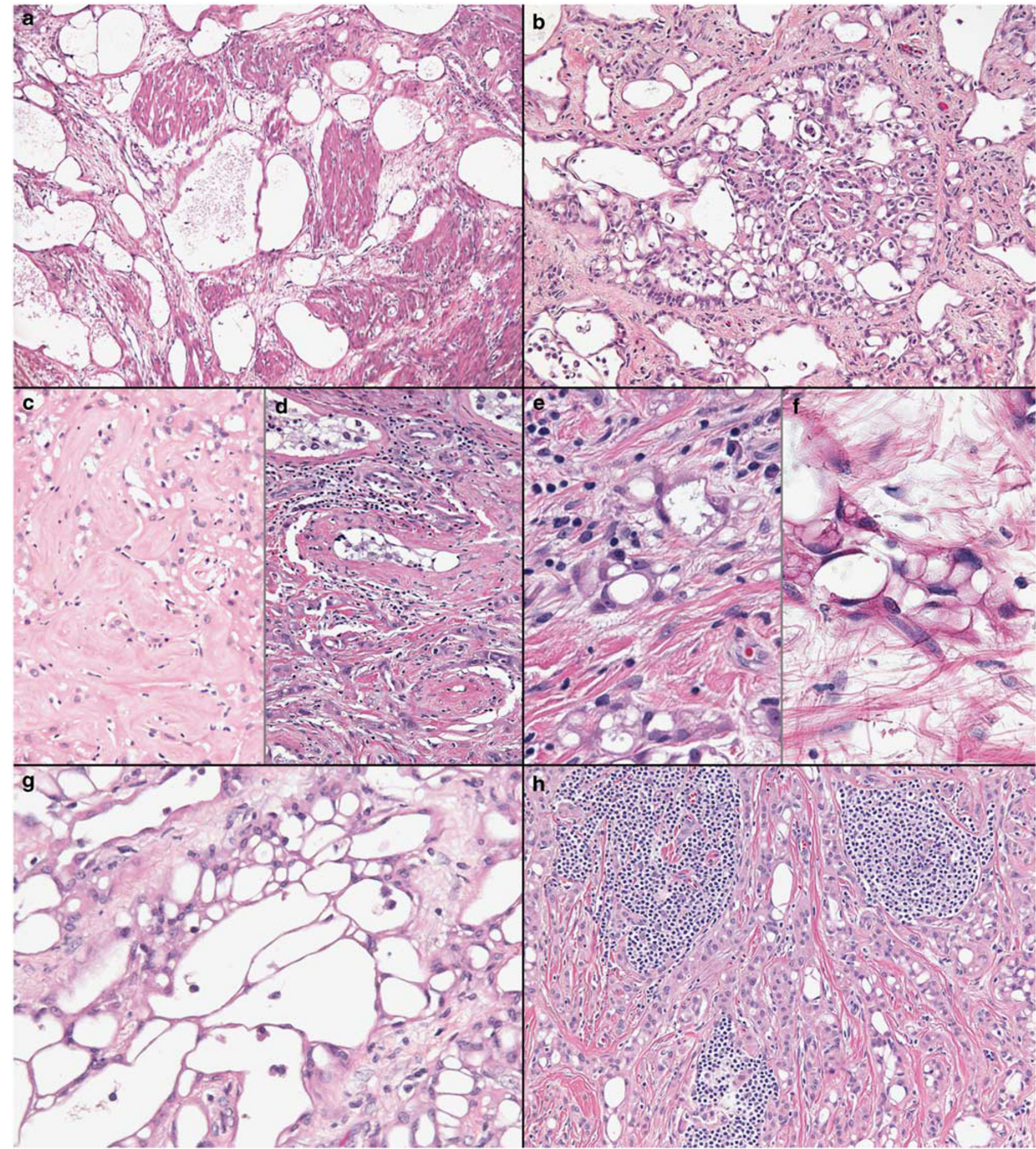

Figure 1 (a) Cystic, angiomatoid adenomatoid tumor within large myometrial bundles often mimics uterine smooth muscle tumors on clinical grounds $(\mathrm{H} \& \mathrm{E}, \times 100)$. (b) Dense glandular or solid patterns of testicular adenomatoid tumor can mimic yolk sac or sex-cord stromal tumors $(\mathrm{H} \& \mathrm{E}, \times 200)$. (c) Paratesticular hyalinization and (d) infiltration in an epididymal adenomatoid tumor raise the consideration of mesothelioma (H\&E, $\times 400)$. (e) Signet ring and (f) lipoblast-like cells are not uncommon in adenomatoid tumors and can mimic malignancy $(H \& E, \times 400)$. (g) Distinctive thread-like bridging strands crossing tubular spaces, identified in all genital tract adenomatoid tumors are a useful diagnostic feature $(\mathrm{H} \& \mathrm{E}, \times 200)$. (h) Lymphoid aggregates are useful diagnostic features of male genital tract adenomatoid tumors, but are infrequent in female genital tract sites (H\&E, $\times 100)$.

or uterine endometrial carcinoma. Among the female genital tract adenomatoid tumors not identified incidentally, presumed clinical diagnoses included leiomyomata, adenomyosis, endometriosis (myometrial adenomatoid tumors), or an unspecified mass lesion (ovarian adenomatoid tumor). 
Table 4 Immunohistochemical results of female and male genital tract adenomatoid tumors

\begin{tabular}{|c|c|c|c|c|c|c|}
\hline Origin & CKAE1/CAM5.2 (M/C) & Calretinin (N/C) & $D 2-40(N / C)$ & $W T-1(N)$ & CK5/6 (C) & Caldesmon $(N / C)$ \\
\hline Female genital tract & $\begin{array}{c}32 / 32(100 \%) \\
\mathrm{ME}=3 \\
\mathrm{MI}=2.9\end{array}$ & $\begin{array}{c}32 / 32(100 \%) \\
M E=2.8 \\
M I=2.5\end{array}$ & $\begin{array}{c}32 / 32(100 \%) \\
\mathrm{ME}=2.8 \\
\mathrm{MI}=2.5\end{array}$ & $\begin{array}{c}31 / 32(97 \%) \\
M E=2.1 \\
M I=1.5\end{array}$ & $\begin{array}{c}5 / 32(16 \%) \\
\mathrm{ME}=1.6 \\
\mathrm{MI}=1\end{array}$ & $\begin{array}{c}1 / 32(3 \%) \\
M E=3 \\
M I=2\end{array}$ \\
\hline Male genital tract & $\begin{array}{c}12 / 12(100 \%) \\
\mathrm{ME}=3 \\
\mathrm{MI}=3\end{array}$ & $\begin{array}{c}12 / 12(100 \%) \\
\mathrm{ME}=2.9 \\
\mathrm{MI}=2.9\end{array}$ & $\begin{array}{c}12 / 12(100 \%) \\
M E=2.7 \\
M I=3\end{array}$ & $\begin{array}{c}11 / 12(92 \%) \\
M E=2.7 \\
M I=1.7\end{array}$ & $\begin{array}{c}1 / 12(8 \%) \\
M E=1 \\
M I=1\end{array}$ & $0 / 12(0 \%)$ \\
\hline
\end{tabular}

M, membranous; C, cytoplasmic; N, nuclear; ME, mean extent; MI, mean intensity.

Follow-up for all 44 genital tract tumors showed no evidence of recurrence or disease progression (mean $=95.5$ months, range $=2-155$ months).

\section{Morphologic Features}

Comparative histologic features of female and male genital tract adenomatoid tumors are listed in Table 3. Histologic patterns identified in both female and male genital tract adenomatoid tumors included adenoid, angiomatoid, cystic, glandular, oncocytic, solid, and tubular (Figures 1a and b). Tumoral infarction was identified in 4 of $12(25 \%)$ and in 1 of $32(3 \%)$ male and female adenomatoid tumors, respectively, with all 5 of the cases demonstrating mild to moderate cytologic atypia. Two of the four male and the single female adenomatoid tumor with infarction also demonstrated prominent stromal hyalinization and/or myofibroblastic proliferation (Figure 1c). Although extra-organ extension, marked cytologic atypia, tumor cell necrosis, or mitotic figures were not seen in any of the cases, an infiltrative pattern of growth was common $(75 \%$ overall incidence) to both male and female genital tract tumors (Figure 1d). Fallopian tube adenomatoid tumors, all identified as incidental histologic findings, demonstrated a well-circumscribed border without an infiltrative pattern. The single, incidentally discovered, ovarian adenomatoid tumor lacked an invasive growth pattern. Signet-ring cell morphology was identified in all male and in $94 \%$ of female adenomatoid tumors, and was focal to moderate in extent (Figure 1e). Vacuolated, lipoblast-like cells were also a frequent finding in both male and female genital tract tumors, ranging from focal to moderate in extent (Figure 1f). Distinctive thread-like bridging strands crossing tubular spaces were present in all genital tract adenomatoid tumors (Figure 1g), although focal in some cases. Lymphoid aggregates, either admixed with tumor cells or around the periphery, were seen in all male genital tract adenomatoid tumors ranging from focal to moderate in extent (Figure 1h). Among the female genital tract sites, lymphoid aggregates were infrequent $(13 \%)$ and, when present, were focal.

\section{Immunohistochemical Results}

Immunohistochemical results for female and male genital tract tumors using antibodies pankeratin, calretinin, D2-40, WT-1, CK5/6, and caldesmon are listed in Table 4. Diffuse, strong membranous and cytoplasmic reactivity with pankeratin (CKAE1/ CAM5.2) was identified among all adenomatoid tumors (Figure 2a). Although positive staining with calretinin (nuclear and cytoplasmic) and D2-40 (nuclear and/or cytoplasmic) was seen in all cases with similar intensity and extent (Figures $2 \mathrm{~b}$ and $\mathrm{c}$ ), background staining was often seen with calretinin (endometrial epithelium and stroma, myometrium in female genital tract tumors; epididymal epithelium and Sertoli cells in male genital tract tumors), making recognition of tumoral staining challenging in certain cases. Similarly, although nuclear WT-1 antibody staining was seen in nearly all (97\%) female genital tract adenomatoid tumors, extensive and often stronger reactivity was seen in endometrial epithelium and myometrium making recognition of tumoral staining difficult. Nuclear reactivity with WT-1 was seen in most (92\%) male genital tract adenomatoid tumors without significant background staining (Figure 2d). Immunostaining with D2-40 was cleaner with additional reactivity in only the lymphatic endothelium (Figure 2c). Cytoplasmic immunoreactivity for CK5/6 was seen in only $5 / 32$ $(16 \%)$ and in $1 / 12$ (8\%) female and male adenomatoid tumors, respectively, and when present was focal and faint (Figure 2e). Only one (3\%) female genital tract adenomatoid tumor demonstrated positive (nuclear and cytoplasmic) staining with caldesmon with moderate intensity and extent, whereas staining was not identified in any of the 12 male genital tract adenomatoid tumors (Figure 2f).

\section{Discussion}

Of the morphologic features evaluated in female and male genital tract adenomatoid tumors, only the presence of lymphoid aggregates (Figure 1h) demonstrated a statistically significant sex predilection. Lymphoid aggregates were present in $100 \%$ of male genital tract tumors, but in only $13 \%$ of female 


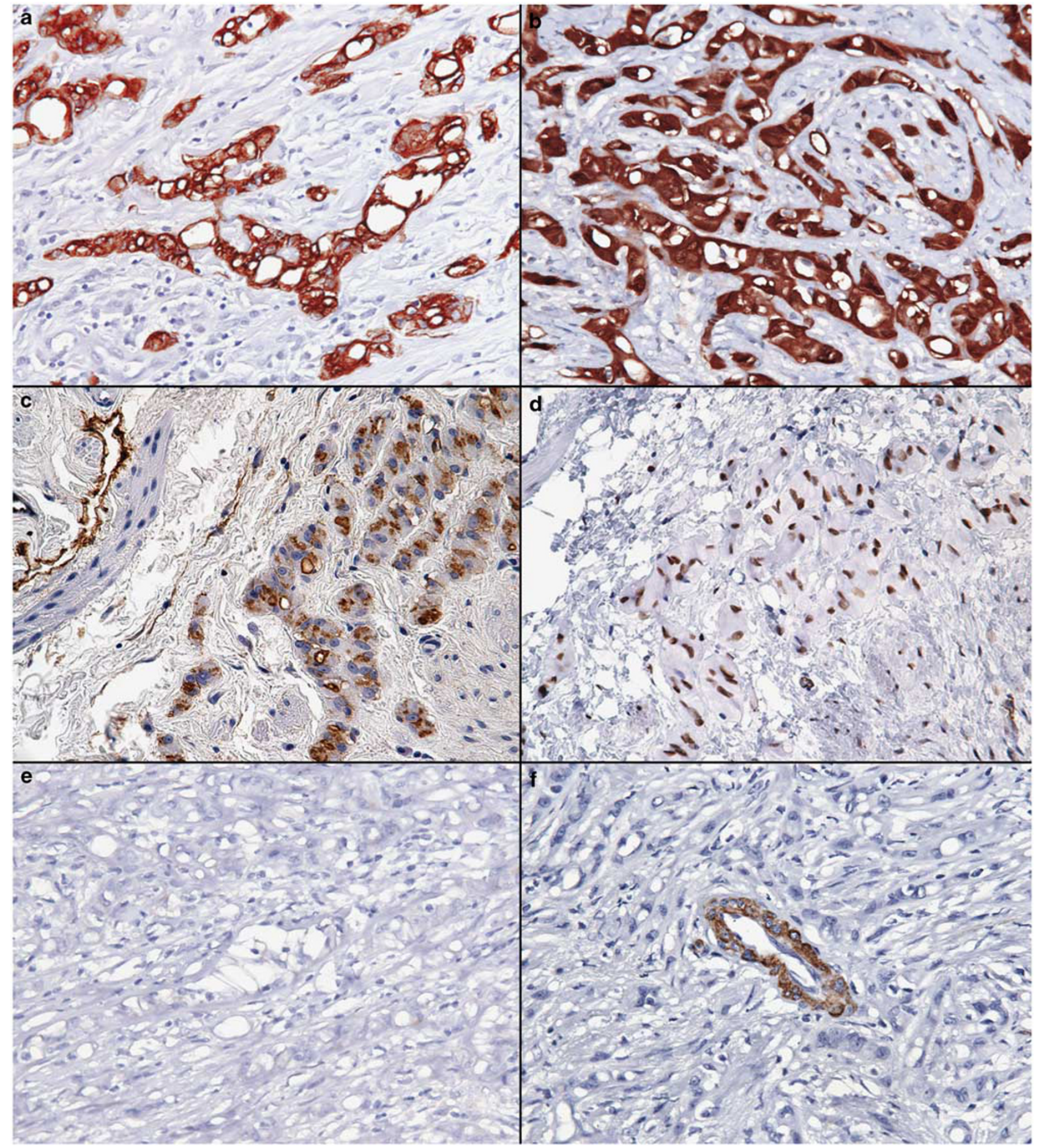

Figure 2 (a) Adenomatoid tumor with diffuse strong pankeratin membranous and cytoplasmic immunoreactivity $(\times 200)$. Although staining with both (b) calretinin and (c) D2-40 demonstrated equally diffuse, strong reactivity in male genital tract adenomatoid tumors (shown here), calretinin showed marked background staining in female sites making accurate tumoral recognition challenging $(\times 200)$. (c) Note positive internal control staining in lymphatics (left). (d) Similarly, although tumoral reactivity for WT-1 antibody in male adenomatoid tumors (shown here) was straightforward, in female sites background staining often precluded accurate detection $(\times 200)$. (e) Most adenomatoid tumors from both male and female sites showed either negative to focal, weak cytoplasmic staining with CK5/6 $(\times 200)$. (f) Staining with caldesmon was essentially negative in all adenomatoid tumors $(\times 400)$. Note positive internal control staining of vascular smooth muscle.

genital tract tumors $(P<0.0001)$, and, when present in female sites, were typically focal. Variably described as follicular inflammatory infiltrates, lymphocyte collections, chronic lymphoid follicles, or lymphoid aggregates (with or without germinal centers), ${ }^{19,22,25,34-36}$ these chronic inflammatory cell 


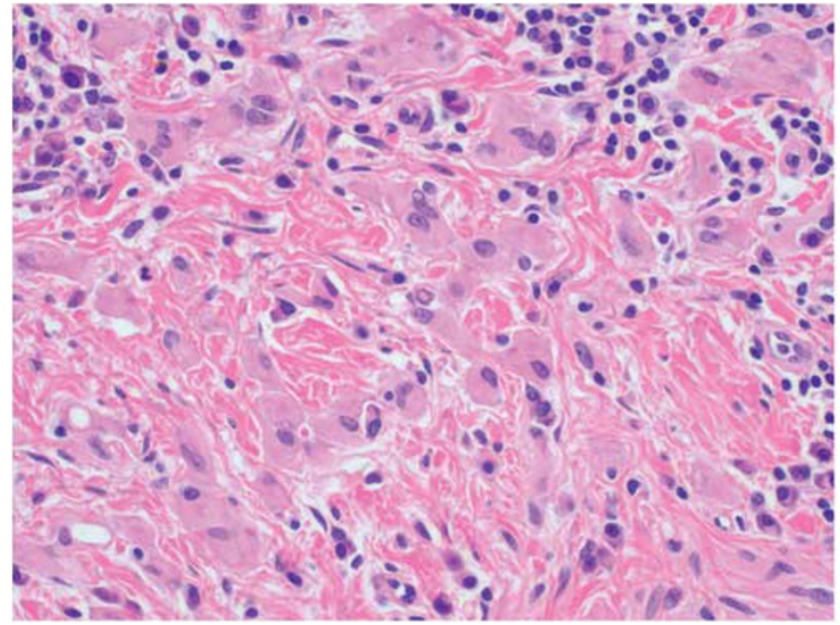

Figure 3 Paratesticular oncocytic adenomatoid tumor predominantly composed of epithelioid tumor cells with abundant granular, eosinophilic cytoplasm admixed with scattered lymphoplasmacytic inflammation $(\mathrm{H} \& \mathrm{E}, \times 600)$. Recognition of this oncocytic pattern is essential in differential diagnostic assessment, which includes sex-cord stromal tumor as well as other nonepithelial/epithelial neoplasms.

clusters/follicles have been reported to be useful diagnostic features of all genital tract adenomatoid tumors $^{36,37}$ despite a lack of formal appraisal across gender and site. Although an initial study in uterine/fallopian tube adenomatoid tumors reported 'inflammation' in up to $80 \%$ of cases, ${ }^{35}$ only one other study to our knowledge has formally evaluated lymphoid aggregates in uterine adenomatoid tumors. In that report, an incidence of $50 \%$ was observed, which is still significantly lower than that observed in male sites. ${ }^{25}$ Although a definitive etiology for gender differences in associated chronic inflammation in adenomatoid tumors is uncertain, we speculate that sites more prone to trauma (testis/ epididymis versus uterus) may account for this disparity. In contrast, the presence of thread-like bridging strands traversing tubular spaces (Figure 1g) appears to be a reproducible corroborative diagnostic feature for genital tract adenomatoid tumors regardless of gender, although it may require diligent search to identify them. ${ }^{36,38}$

A variety of other morphologic patterns, each of which has been previously described in adenomatoid tumors, were also identified in female and male tumors in this series. These include adenoid, angiomatoid, cystic, glandular, solid, and tubular patterns, and when encountered in pure form they may pose diagnostic problems. ${ }^{14,15,26,46}$ Two additional, less recognized morphologic patterns, oncocytic and reactive ischemia, can pose particularly difficult diagnostic problems because of their overlap with other neoplasms. One epididymal and two paratesticular/testicular adenomatoid tumors in this series had nearly pure ( $>95 \%$ ) oncocytic morphology (Figure 3), a pattern similar to the reported ovarian 'oxyphilic' adenomatoid tumors. ${ }^{39}$ When prominent, this cytoplasmic eosinophilia may mi- mic sex-cord stromal tumor or other nonepithelial and epithelial neoplasms, requiring additional diagnostic evaluation. The presence of infarction and prominent stromal hyalinization with or without myofibroblastic proliferation, another potential diagnostic pitfall, ${ }^{40}$ was also seen in three of our genital tract adenomatoid tumors (Figure 1c). When these changes are encountered, they often raise consideration for a malignant process, such as invasive carcinoma or mesothelioma. Immunohistochemical stains will assist in the former, whereas localization to the genital tract site in absence of disease elsewhere in the peritoneum should assist in the latter differential diagnosis. Additionally, a marked angiomatoid pattern in a testicular adenomatoid tumor as well as potential cytokeratin immunoreactivity raises the possibility of epithelioid (histiocytic) hemangioma, ${ }^{41}$ which in most instances can be resolved with additional vascular and mesothelial immmunohistochemical markers.

The proposed histogenesis of genital tract adenomatoid tumors has varied from mesothelial in the early $1940 \mathrm{~s}^{13}$ to a broader spectrum nearly two decades later, which in addition to mesothelial included endothelial, mesonephric, and mullerian. ${ }^{22}$ Since then, both ultrastructural and immunohistochemical studies of male and female genital tract adenomatoid tumors have shown support for a mesothelial origin. ${ }^{12,15-35}$ Of the markers reported to have superior sensitivity in identifying mesothelialassociated neoplasms, ${ }^{42-46}$ calretinin, D2-40, WT-1, CK5/6, and caldesmon were selected to determine immunoreactivity in both male and female genital tract adenomatoid tumors, with pankeratin staining used to corroborate the location and extent of tumor. Although both calretinin and D2-40 demonstrated equally diffuse and strong tumoral immunoreactivity, the significant background staining with calretinin in many cases of female genital tract adenomatoid tumors suggests that D2-40 may be more useful. Similarly, WT-1 antibody also demonstrated background staining in female genital tract sites; it also showed decreased staining intensity compared to either calretinin or D2-40, further limiting its diagnostic utility. Adenomatoid tumor immunoreactivity with $\mathrm{D} 2-40$ is not a novel finding, ${ }^{4-51}$ but this study reports the largest series with immunohistochemical assessment to date.

Cytokeratin 5/6 (CK5/6) is often included as a recommended antibody for adenomatoid tumors, given its well-known reactivity in mesothelialassociated neoplasms. ${ }^{42-46}$ However, there is a paucity of studies exploring its immunoreactivity in extragenital ${ }^{10,52-54}$ or genital adenomatoid tumors. ${ }^{39}$ Moreover, many of these studies report only focal staining with $C K 5 / 6$. We report similar focal, weak staining with CK5/6 in both male and female genital tract adenomatoid tumors, limiting its utility in this setting. Despite the reported sensitivity of caldesmon in mesothelioma, ${ }^{42,43}$ earlier studies have 
not formally evaluated adenomatoid tumors; the extremely low sensitivity of caldesmon in our study $(2 \%)$ indicates that this also is of limited utility in genital tract adenomatoid tumors.

In summary, although lymphoid aggregates are common in adenomatoid tumors of the male genital tract, they are relatively infrequent in female genital sites. With the exception of tubal and, possibly, paraovarian sites, most genital tract adenomatoid tumors exhibit an infiltrative pattern. Thread-like bridging strands are consistently present in all genital tract adenomatoid tumors, and remain useful in diagnosis. Despite their known reactivity in mesothelial lesions, the low sensitivity of CK5/6 and caldesmon limit their diagnostic utility in adenomatoid tumors. Compared to WT-1, CK5/6, and caldesmon, calretinin and D2-40 demonstrate a higher sensitivity in genital tract adenomatoid tumors. D2-40 is the most useful marker for adenomatoid tumors in the female genital tract because of its combined high sensitivity and limited background staining.

\section{References}

1 Adem C, Schneider M, Hoang C. Pathologic quiz case: an unusual umbilical mass. Arch Pathol Lab Med 2003;127:e303-e304.

2 Craig JR, Hart WR. Extragenital adenomatoid tumor: evidence for the mesothelial theory of origin. Cancer 1979;43:1678-1681.

3 Hanada S, Okumura Y, Kaida K. Multicentric adenomatoid tumors involving uterus, ovary, and appendix. J Obstet Gynaecol Res 2003;29:234-238.

4 Hanrahan JB. A combined papillary mesothelioma and adenomatoid tumor of the omentum; report of a case. Cancer 1963;16:1497-1500.

5 Hayes SJ, Clark P, Mathias R, et al. Multiple adenomatoid tumours in the liver and peritoneum. J Clin Pathol 2007;60:722-724.

6 Isotalo PA, Nascimento AG, Trastek VF, et al. Extragenital adenomatoid tumor of a mediastinal lymph node. Mayo Clin Proc 2003;78:350-354.

7 Kaplan MA, Tazelaar HD, Hayashi T, et al. Adenomatoid tumors of the pleura. Am J Surg Pathol 1996;20:1219-1223.

8 Natarajan S, Luthringer DJ, Fishbein MC. Adenomatoid tumor of the heart: report of a case. Am J Surg Pathol 1997;21:1378-1380.

9 Overstreet K, Wixom C, Shabaik A, et al. Adenomatoid tumor of the pancreas: a case report with comparison of histology and aspiration cytology. Mod Pathol 2003;16:613-617.

10 Plaza JA, Dominguez F, Suster S. Cystic adenomatoid tumor of the mediastinum. Am J Surg Pathol 2004;28:132-138.

11 Simpson PR. Adenomatoid tumor of the adrenal gland. Arch Pathol Lab Med 1990;114:725-727.

12 Stephenson TJ, Mills PM. Adenomatoid tumours: an immunohistochemical and ultrastructural appraisal of their histogenesis. J Pathol 1986;148:327-335.

13 Masson P RJ, Simard L. Le mesotheliome benin de la sphere genitale. Rev Can Biol 1942;1:720-751.
14 Golden A, Ash J. Adenomatoid tumors of the genital tract. Am J Pathol 1945;21:63-79.

15 Barwick KW, Madri JA. An immunohistochemical study of adenomatoid tumors utilizing keratin and factor VIII antibodies. Evidence for a mesothelial origin. Lab Invest 1982;47:276-280.

16 Davy CL, Tang CK. Are all adenomatoid tumors adenomatoid mesotheliomas? Hum Pathol 1981; 12:360-369.

17 Delahunt B, Eble JN, King D, et al. Immunohistochemical evidence for mesothelial origin of paratesticular adenomatoid tumour. Histopathology 2000;36: 109-115.

18 Delahunt B EJ, Srigley JR, et al. Paratesticular adenomatoid tumor: assessment of calretinin immunoexpression and cell proliferation indices. J Urol Pathol 2000;12:105-115.

19 Ferenczy A, Fenoglio J, Richart RM. Observations on benign mesothelioma of the genital tract (adenomatoid tumor). A comparative ultrastructural study. Cancer 1972;30:244-260.

20 Goddard MJ, Grant JW. Adenomatoid tumours: a mucin histochemical and immunohistochemical study. Histopathology 1992;20:57-61.

21 Huang CC, Chang DY, Chen CK, et al. Adenomatoid tumor of the female genital tract. Int J Gynaecol Obstet 1995;50:275-280.

22 Jackson JR. The histogenesis of the adenomatoid tumor of the genital tract. Cancer 1958;11:337-350.

23 Mai KT, Yazdi HM, Perkins DG, et al. Adenomatoid tumor of the genital tract: evidence of mesenchymal cell origin. Pathol Res Pract 1999;195:605-610.

24 Marcus JB, Lynn JA. Ultrastructural comparison of an adenomatoid tumor, lymphangioma, hemangioma, and mesothelioma. Cancer 1970;25:171-175.

25 Nogales FF, Isaac MA, Hardisson D, et al. Adenomatoid tumors of the uterus: an analysis of 60 cases. Int J Gynecol Pathol 2002;21:34-40.

26 Otis CN. Uterine adenomatoid tumors: immunohistochemical characteristics with emphasis on Ber-EP4 immunoreactivity and distinction from adenocarcinoma. Int J Gynecol Pathol 1996;15:146-151.

27 Quigley JC, Hart WR. Adenomatoid tumors of the uterus. Am J Clin Pathol 1981;76:627-635.

28 Said JW, Nash G, Lee M. Immunoperoxidase localization of keratin proteins, carcinoembryonic antigen, and factor VIII in adenomatoid tumors: evidence for a mesothelial derivation. Hum Pathol 1982;13: 1106-1108.

29 Salazar H, Kanbour A, Burgess F. Ultrastructure and observations on the histogenesis of mesotheliomas, 'adenomatoid tumors', of the female genital tract. Cancer 1972;29:141-152.

30 Schwartz EJ, Longacre TA. Adenomatoid tumors of the female and male genital tracts express WT1. Int J Gynecol Pathol 2004;23:123-128.

31 Srigley JR, Colgan TJ. Multifocal and diffuse adenomatoid tumor involving uterus and fallopian tube. Ultrastruct Pathol 1988;12:351-355.

32 Taxy JB, Battifora H, Oyasu R. Adenomatoid tumors: a light microscopic, histochemical, and ultrastructural study. Cancer 1974;34:306-316.

33 Tiltman AJ. Adenomatoid tumours of the uterus. Histopathology 1980;4:437-443.

34 Young RH, Silva EG, Scully RE. Ovarian and juxtaovarian adenomatoid tumors: a report of six cases. Int J Gynecol Pathol 1991;10:364-371. 
35 Youngs LA, Taylor HB. Adenomatoid tumors of the uterus and fallopian tube. Am J Clin Pathol 1967;48:537-545.

36 Amin MB. Selected other problematic testicular and paratesticular lesions: rete testis neoplasms and pseudotumors, mesothelial lesions and secondary tumors. Mod Pathol 2005;18(Suppl 2):S131-S145.

37 Young RH. Testicular tumors-some new and a few perennial problems. Arch Pathol Lab Med 2008;132: 548-564.

38 Hes O, Perez-Montiel DM, Alvarado Cabrero I, et al. Thread-like bridging strands: a morphologic feature present in all adenomatoid tumors. Ann Diagn Pathol 2003;7:273-277.

39 Phillips V, McCluggage WG, Young RH. Oxyphilic adenomatoid tumor of the ovary: a case report with discussion of the differential diagnosis of ovarian tumors with vacuoles and related spaces. Int J Gynecol Pathol 2007;26:16-20.

40 Skinnider BF, Young RH. Infarcted adenomatoid tumor: a report of five cases of a facet of a benign neoplasm that may cause diagnostic difficulty. Am J Surg Pathol 2004;28:77-83.

41 Banks ER, Mills SE. Histiocytoid (epithelioid) hemangioma of the testis. The so-called vascular variant of 'adenomatoid tumor'. Am J Surg Pathol 1990;14:584-589.

42 Comin CE, Dini S, Novelli L, et al. h-Caldesmon, a useful positive marker in the diagnosis of pleural malignant mesothelioma, epithelioid type. Am J Surg Pathol 2006;30:463-469.

43 Comin CE, Saieva C, Messerini L. h-Caldesmon, calretinin, estrogen receptor, and Ber-EP4: a useful combination of immunohistochemical markers for differentiating epithelioid peritoneal mesothelioma from serous papillary carcinoma of the ovary. Am J Surg Pathol 2007;31:1139-1148.
44 Marchevsky AM. Application of immunohistochemistry to the diagnosis of malignant mesothelioma. Arch Pathol Lab Med 2008;132:397-401.

45 Ordonez NG. What are the current best immunohistochemical markers for the diagnosis of epithelioid mesothelioma? A review and update. Hum Pathol 2007;38:1-16.

46 Yaziji H, Battifora H, Barry TS, et al. Evaluation of 12 antibodies for distinguishing epithelioid mesothelioma from adenocarcinoma: identification of a three-antibody immunohistochemical panel with maximal sensitivity and specificity. Mod Pathol 2006;19:514-523.

47 Ghorab Z, Khalifa MA, Kahn HJ. Adenomatoid tumors of the female and male genital tract express D2-40. Mod Pathol 2006;632:138A.

48 Hamamatsu A, Arai T, Iwamoto M, et al. Adenomatoid tumor of the adrenal gland: case report with immunohistochemical study. Pathol Int 2005;55:665-669.

49 Kuroda N, Toi M, Hiroi M, et al. Diagnostic pitfall of D2-40 in adenomatosid tumour. Histopathology 2007;51:719-721.

50 Nagata S, Aishima S, Fukuzawa K, et al. Adenomatoid tumour of the liver. J Clin Pathol 2008;61:777-780.

51 Ordonez NG. D2-40 and podoplanin are highly specific and sensitive immunohistochemical markers of epithelioid malignant mesothelioma. Hum Pathol 2005;36:372-380.

52 Garg K, Lee P, Ro JY, et al. Adenomatoid tumor of the adrenal gland: a clinicopathologic study of 3 cases. Ann Diagn Pathol 2005;9:11-15.

53 Isotalo PA, Keeney GL, Sebo TJ, et al. Adenomatoid tumor of the adrenal gland: a clinicopathologic study of five cases and review of the literature. Am J Surg Pathol 2003;27:969-977.

54 Yeh CJ, Chuang WY, Chou HH, et al. Multiple extragenital adenomatoid tumors in the mesocolon and omentum. APMIS 2008;116:1016-1019. 